\title{
A REMARK ON CURVES COVERED BY COVERINGS
}

\author{
MARC COPPENS
}

(Communicated by Louis J. Ratliff, Jr.)

\begin{abstract}
Let $f: C^{\prime} \rightarrow C$ be a covering between two smooth irreducible projective curves. Let $p$ be a prime number. If $C^{\prime}$ is a covering of degree $p$ of a curve of genus $h$ and if $g(C) \geq\left(\begin{array}{c}p \\ 2\end{array}\right)(h+3)+h+3-2 p$, then $C$ is a covering of degree $p$ of a curve of genus at most $h$.
\end{abstract}

Let $C$ be a smooth irreducible projective curve of genus $g$ defined over $\mathbb{C}$. Definition. We say that $C$ is of type $(d ; h)$ if there exists a covering $\pi: C \rightarrow E$ with $\operatorname{deg}(\pi)=d$ and $g(E) \leq h$.

Statement $S(d ; h ; g)$. If $f: C^{\prime} \rightarrow C$ is a morphism with $g(C)=g$ and if $C^{\prime}$ is of type $(d ; h)$, then $C$ is of type $(d ; h)$.

Statement $S(d ; h ; g)$ is proved for the cases $d=2$ (see [1,2]), $d=3$ (see [1]), and $d=4, g \neq 7$ (see [1]). For $h=0$ it is almost trivial (see, e.g., [4]).

In this paper we prove

Theorem. Let $p$ be a prime number. Statement $S(p ; h ; g)$ holds for $g \geq$ $\left(\begin{array}{l}p \\ 2\end{array}\right)(h+3)+h+3-2 p$.

Remarks (from the referee). (1) There is an important difference between Statement $S(d ; h ; g)$ in this paper and in [1]. As a matter of fact, in [1] a curve $C$ is said to be of type $(d ; h)$ if $\operatorname{deg}(\pi) \leq d$ (inequality instead of equality).

(2) The referee informed me about D. Abramovich's Ph.D. Thesis, Subvarieties of Abelian varieties and of jacobians of curves, of which Abramovich sent me a copy. Amongst a lot of other interesting results, it contains again results concerning the topic studied in this paper. Also, from a letter, it is clear to me that he knows our theorem already.

If $d$ is not a prime number then the situation is more complicated. Assume $d=n=a n^{\prime}$ for some $a, n^{\prime} \in \mathbb{Z}_{\geq 2}$ and assume there exists a diagram

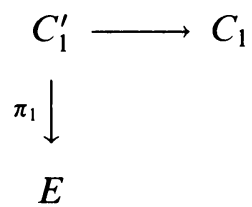

Received by the editors August 20, 1991 and, in revised form, October 28, 1991.

1991 Mathematics Subject Classification. Primary 14H45; Secondary 14H55.

The author is affiliated with the University at Leuven (Celestijnenlaan 200B B 3030 Leuven Belgium) as a research fellow. 
with $\operatorname{deg}\left(\pi_{1}\right)=n^{\prime}, g(E)=h$, and $g\left(C_{1}\right)=g_{1}$ such that $C_{1}$ is not of type $\left(n^{\prime \prime} ; h\right)$ for some $n^{\prime \prime} \leq n^{\prime}$. Take a covering $\tau: C \rightarrow C_{1}$ of degree $a$. Assume there exists a covering $\mu: C \rightarrow E_{1}$ with $\operatorname{deg}(\mu) \leq n$ and $g\left(E_{1}\right) \leq h$. Consider $(\tau ; \mu): C \rightarrow C_{1} \times E_{1}$, and let $C^{\prime \prime}$ be the normalization of the image of $(\tau ; \mu)$. One obtains a diagram of coverings

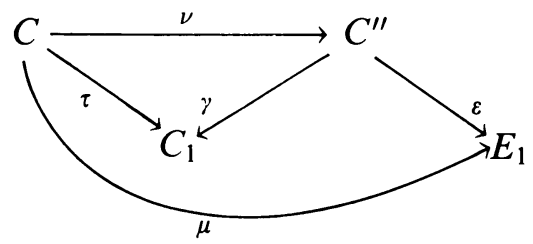

It is well known (see, e.g., $\left[3, \S 1\right.$, (iii)]) that $g\left(C^{\prime \prime}\right) \leq(a-1)(n-1)+a g_{1}+n h$. So if we take $g(C)$ large enough then $\nu$ is nontrivial. Also $\gamma$ is nontrivial, otherwise $\varepsilon$ gives us that $C_{1}$ is of type $\left(n^{\prime \prime} ; h\right)$ for some $n^{\prime \prime} \leq n^{\prime}$. Choosing suited ramification in the covering $\tau$ (e.g., a fibre of type $(a-1) Q_{1}+Q_{2}$ suffices), this becomes impossible. Let $\widetilde{C^{\prime}}$ be the normalization of an irreducible component of $C_{1}^{\prime} \times{ }_{C_{1}} C$. Taking a covering $C^{\prime} \rightarrow \widetilde{C}^{\prime}$ if necessary, we obtain the following situation

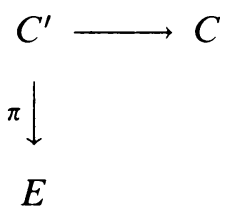

with $\operatorname{deg}(\pi)=n, g(E) \leq h$, but $C$ is not of type $(n ; h)$. Moreover, we can take $g(C)$ arbitrarily large.

In [1] Abramovich and Harris prove the existence of a diagram

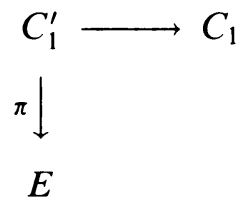

with $g\left(C_{1}\right)=5, \operatorname{deg}(\pi)=3, g(E)=2$, and $C_{1}$ is not of type $\left(n^{\prime \prime} ; 2\right)$ for $n^{\prime \prime}=2$ or 3 . Hence for each $a \in \mathbb{Z}_{\geq 2}$ there exists no $g_{0}$ such that $S(3 a ; 2 ; g)$ holds for $g \geq g_{0}$.

Proof of the theorem. Consider the situation

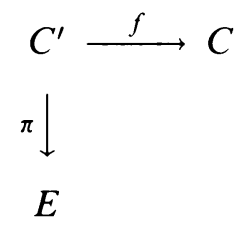

with $\operatorname{deg}(\pi)=p, g(E)=h$, and $g(C)=g \geq\left(\begin{array}{l}p \\ 2\end{array}\right)(h+3)+h+3-2 p$. For $n \in \mathbb{Z}_{\geq 2}, D \in E^{(h+n)}$, and $P \in E$ general, the linear systems $|D-P|$ and $|D|$ on $E$ are without fixed points. Using the fact that $f_{*}$ preserves linear equivalence (see [4]) also $L:=\left|f_{*} \pi^{*}(D)\right|$ and $\left|L-f_{*} \pi^{*}(P)\right|=\left|f_{*} \pi^{*}(D-P)\right|$ have no fixed points on $C$. In particular,

$$
\operatorname{dim}(L) \geq \operatorname{dim}\left(\left|L-f_{*} \pi^{*}(P)\right|\right)+1 .
$$


Assume we have equality in $(*)$. Let $\phi: C \rightarrow \mathbb{P}^{r}$ be the morphism associated to $L$, let $C_{1}$ be the normalization of $\phi(C)$, and let $\rho: C \rightarrow C_{1}$ be the induced covering. Equality in $(*)$ implies that $\operatorname{deg}(\rho)=p$ and for each $P \in E$ the divisor $f_{*} \pi^{*}(P)$ is a fibre of $\rho$. It follows that the morphism $(\pi ; \rho \circ f): C^{\prime} \rightarrow$ $E \times C_{1}$ factors through $E$. Hence $E$ dominates $C_{1}$, so $g\left(C_{1}\right) \leq h$. This implies that $C$ is of type $(p ; h)$.

So now, assume we have

$$
\operatorname{dim}(L) \geq \operatorname{dim}\left(\left|L-f_{*} \pi^{*}(P)\right|\right)+2 .
$$

Because $\operatorname{deg}(\rho)<p$ now, we have that $\rho$ is an isomorphism (here we use that $p$ is a prime number). If $p=2$ then for $D \in E^{(h+1)}$ general one finds that $\operatorname{dim}\left(\left|f_{*} \pi^{*}(D)+f_{*} \pi^{*}(P)\right|\right) \geq \operatorname{dim}\left(\left|f_{*} \pi^{*}(D)\right|\right)+\operatorname{deg}\left(f_{*} \pi^{*}(P)\right)$. This implies that $\left|f_{*} \pi^{*}(D)\right|$ is nonspecial, hence $1 \leq 2 h+2-g$, i.e., $g \leq 2 h+1$. Since $g>2 h+1$, we obtain a contradiction. For $p \neq 2$ we are going to use Castelnuovo theory in order to obtain a similar situation. The use of Castelnuovo theory is inspired by $\S 3$ in [1].

Take $D_{1} \in E^{(h+2)}$ general and $D_{2} \in E^{(h+n)} \quad(n \geq 2)$ general. Take $P \in E$ general and write $f_{*} \pi^{*}(P)=P_{1}+P_{2}+\cdots+P_{p}$. Since $L_{i}=\left|f_{*} \pi^{*}\left(D_{i}\right)\right|$ is simple, we obtain that there exists $E_{i} \in L_{i}$ with $\operatorname{cd}\left(E_{i} ; f_{*} \pi^{*}(P)\right)=P_{i}$. (For two effective divisors $E_{1}$ and $E_{2}$ on $C$, one has $E_{0}=\operatorname{cd}\left(E_{1} ; E_{2}\right)$ if $E_{i} \geq E_{0}$ and $\operatorname{Supp}\left(E_{1}-E_{0}\right) \cap \operatorname{Supp}\left(E_{2}-E_{0}\right)=\varnothing$.) Consider $\left|E_{1}+E_{2}+P_{3}+\cdots+P_{p}\right| \supset$ $\left(\left|E_{1}+E_{2}\right|+P_{3}+\cdots+P_{p}\right) \cup\left(\left|E_{1}+P_{2}+\cdots+P_{p}\right|+\left(E_{2}-P_{2}\right)\right)$. Also $\left|E_{1}+P_{2}+\cdots+P_{p}\right|=$ $\left|f_{*} \pi^{*}\left(D_{1}+P\right)-P_{1}\right|$ has no fixed points, so $\left|E_{1}+E_{2}+P_{3}+\cdots+P_{p}\right|$ has no fixed points. Also $\left|E_{1}+E_{2}+P_{2}+\cdots+P_{p}\right| \supset\left|E_{2}+P_{1}+\cdots+P_{p}\right|+\left(E_{1}-P_{1}\right)$, hence $\left|E_{1}+E_{2}+P_{2}+\cdots+P_{p}\right|$ has no fixed points. Finally $\left|E_{1}+E_{2}+P_{1}+\cdots+P_{p}\right|=$ $\left|f_{*} \pi^{*}\left(D_{1}+D_{2}+P\right)\right|$ has no fixed points. This implies, for $D \in E^{(2 h+4+n)} \quad(n \geq 0)$ and $P \in E$ general, that one has

$$
\operatorname{dim}\left|f_{*} \pi^{*}(D+P)\right| \geq \operatorname{dim}\left|f_{*} \pi^{*}(D)\right|+3
$$

there exist $E_{i} \in\left|f_{*} \pi^{*}(D+P)\right|$ for $i=1,2$

with $\operatorname{cd}\left(E_{1} ; f_{*} \pi^{*}(P)\right)=P_{1}, \operatorname{cd}\left(E_{2} ; f_{*} \pi^{*}(P)\right)=P_{1}+P_{2}$.

Assume $p>3$. Take $D_{1} \in E^{(2 h+5)}$ general, $D_{2} \in E^{(h+2)}$ general, and $P \in E$ general, and write $f_{*} \pi^{*}(P)=P_{1}+\cdots+P_{p}$. Take $E_{1}, E_{2} \in\left|f_{*} \pi^{*}\left(D_{1}\right)\right|$ with $\operatorname{cd}\left(E_{1} ; f_{*} \pi^{*}(P)\right)=P_{1}, \operatorname{cd}\left(E_{2} ; f_{*} \pi^{*}(P)\right)=P_{1}+P_{2}$, and take $E_{3} \in\left|f_{*} \pi^{*}\left(D_{2}\right)\right|$ with $\operatorname{cd}\left(E_{3} ; f_{*} \pi^{*}(P)\right)=P_{3}$. As before, for $D \in E^{(3 h+7)}$ and $P \in E$ general, one has

$$
\begin{aligned}
& \operatorname{dim}\left|f_{*} \pi^{*}(D+P)\right| \geq \operatorname{dim}\left|f_{*} \pi^{*}(D)\right|+4 ; \\
& \text { there exist } E_{i} \in\left|f_{*} \pi^{*}(D+P)\right| \text { for } i=1,2,3 \\
& \text { with } \operatorname{cd}\left(E_{i} ; f_{*} \pi^{*}(P)\right)=P_{1}+\cdots+P_{i} .
\end{aligned}
$$

Continuing in this way, one finds for $D \in E^{((p-1)(h+3)-2)}$ and $P \in E$ general that

$$
\operatorname{dim}\left|f_{*} \pi^{*}(D+P)\right| \geq \operatorname{dim}\left|f_{*} \pi^{*}(D)\right|+p
$$

This is only possible if $\left|f_{*} \pi^{*}(D)\right|$ is nonspecial on $C$. But $\operatorname{deg}\left(f_{*} \pi^{*}(D)\right)=$ $p(p-1)(h+3)-2 p$ and $\operatorname{dim}\left(\left|f_{*} \pi^{*}(D)\right|\right) \geq 1+2(h+3)+3(h+3)+\cdots+$ $(p-1)(h+3)=\left(\begin{array}{l}p \\ 2\end{array}\right)(h+3)-h-2$. So $\left(\begin{array}{l}p \\ 2\end{array}\right)(h+3)-h-2 \leq p(p-1)(h+3)-2 p-g$, hence $g \leq\left(\begin{array}{c}p \\ 2\end{array}\right)(h+3)-2 p+h+2$. This gives us a contradiction. 
Remark. Using the arguments of [1], the situation is related to the existence of an abelian variety $A$ of dimension $h$ contained in $C^{(p h)}$. Then Theorem 2 in [1] gives us a bound of order $p^{2} h^{2}$, which is worse than ours.

\section{REFERENCES}

1. D. Abramovich and J. Harris, Abelian varieties and curves in $W_{d}(C)$, Compositio Math. 78 (1991), 227-238.

2. J. Harris and J. Silverman, Bi-elliptic curves and symmetric products, Proc. Amer. Math. Soc. 112 (1991), 347-356.

3. G. Martens, Funktionen von vorgegebener Ordnung auf komlexen Kurven, J. Reine Angew. Math. 320 (1980), 68-85.

4. H. Martens, A remark on Abel's Theorem and the mapping of linear series, Comment. Math. Helv. 52 (1977), 557-559.

Katholieke Industriële Hogeschool der Kempen Campus H. I. Kempen, KleinhoefSTRAAT 4, B 2440 GEel, BELgIUM

E-mail address: fgaba11@cc1.kuleuven.ac.be 